\title{
Expression of SATB1 protein in the ductal breast carcinoma tissue microarrays — preliminary study
}

\author{
Christopher Kobierzycki ${ }^{1}$, Andrzej Wojnar ${ }^{2}$, Piotr Dziegiel ${ }^{1,3}$ \\ ${ }^{1}$ Department of Histology and Embryology, Wroclaw Medical University, Wroclaw, Poland \\ ${ }^{2}$ Department of Pathomorphology, Lower Silesian Oncology Centre, Wroclaw, Poland \\ ${ }^{3}$ Department of Physiotherapy, University School of Physical Education, Wroclaw, Poland
}

\begin{abstract}
Special AT-rich sequence-binding protein 1 (SATB1) is a nuclear matrix protein which interacts with specific regions of DNA, ensuring its proper organization and function in the cell. The expression of SATB1 was primarily found in thymocytes, but its increased levels were observed in various types of cancers. However, the knowledge of the function and application possibilities of this protein is still limited. The aim of this study was to investigate the expression of SATB1 protein using immunohistochemistry and tissue microarray (TMA) technique and determine its possible relationship with the proliferative marker Ki-67, estrogen $\alpha$ (ER) and progesterone (PR) receptors as well as grade of histological malignancy $(\mathrm{G})$. The study was performed on material of 48 archival invasive ductal breast cancers (IDC). The TMAs were prepared with the use of $0.6 \mathrm{~mm}$ diameter punches. Immunohistochemical reactions were carried out using antibodies against Ki-67, ER, PR and SATB1 proteins. The intensity of the nuclear reaction was evaluated using a light microscope and computer-assisted image analysis. Expression of Ki-67 and SATB1 protein was observed in $89.58 \%$ and $31.25 \%$ of cancer cases, respectively. $62.5 \%$ of tumors were classified as ER-positive, and $47.92 \%$ as PR-positive. Statistical analysis showed a moderate positive correlation between Ki-67 and SATB1 expression $(\mathrm{r}=0.291, p=0.045$ independently on the receptor status, and $\mathrm{r}=0.392, p=0.032$ in ER-negative tumors). The expression of the Ki-67 antigen increased with higher grade of histological malignancy $(G)$. The results suggest that SATB1 protein may play an indirect role in the cell proliferation and should be evaluated in relation to the other markers. Further studies concerning determination of its role in cancer progression and metastasis, in terms of application as therapeutic target and prognostic marker, are recommended. (Folia Histochemica et Cytobiologica 2013, Vol. 51, No. 4, 333-338)
\end{abstract}

Key words: breast cancer; SATB1; tissue microarrays; Ki-67; ER; PR

\section{Introduction}

Malignant transformation of cells is associated with changes in gene expression. Gross alterations in chromatin organization may be involved in many gene dysregulations, as well as the involvement of specific transcription factors $[1,2]$. Specialized genomic DNA segments that exhibit high affinity to the nuclear matrix in vitro have been designated as matrix/scaffold attachment regions (MARs/SARs).

Correspondence address: C. Kobierzycki, Department of Histology and Embryology, Wroclaw Medical University Chalubinskiego St. 6a, 50-368 Wroclaw, Poland

tel.: +48 7178416 82, fax: +48 7178400 82;

e-mail: christopher.kobierzycki@umed.wroc.pl
They have been proposed to create a unique nuclear microenvironment rich in regulatory proteins able to sustain DNA transcription, replication, repair, and recombination $[3,4]$. During investigations on potential mechanisms that could lead to significant alterations in gene expression in cancer cells a few important proteins with nuclear localization have been identified. Many of these proteins are dramatically up-regulated in human malignancies. They provide not only an architectural core but also recruit functional multi-molecular complexes at the base of chromatin loops to affect multiple distant genes $[5,6]$. Experimental strategies by which these proteins can be exploited as carcinoma-specific diagnostic markers and as targets for antineoplastic therapy have been discussed [3]. 
Special AT-rich sequence binding protein 1 (SATB1) is a genome organizer protein that facilitates various intracellular processes, providing a nuclear architectural platform that binds hundreds of genes, through its interaction with specific genomic sequences; this activity allows expression of these genes to be regulated in parallel, and, thus, to alter cell functions [7]. SATB1 as a MAR-binding protein regulates the genes by folding chromatin into loop domain [8]. It was found to regulate gene expression in thymocytes and pre B-cells [9], but recent studies have shown that SATB1 promotes tumor growth and metastasis through chromatin gene recombination in many neoplasms such as breast, gastrointestinal tract (predominantly colorectal region), liver, laryngeal, lung, thyroid, urinary bladder, ovarian and prostate cancers, melanomas, osteosarcomas, gliomas and some types of leukemias [10-26]. Furthermore, except SATB1 involvement in tumor progression by its mRNA and protein overexpression in some cancers, e.g. in colorectal cancer, significantly lower protein levels were found to be a marker of poor prognosis $[12,27,28]$. All of these findings strongly imply that $S A T B 1$ is a possible candidate oncogene that may contribute to carcinogenesis.

The majority of studies on SATB1 expression in breast cancer have been based on mRNA testing [29], while the use of immunohistochemical method has been rather limited [30]. Moreover, it seems that the immunohistochemistry performed on tissue microarrays (TMA-IHC method) should be abundantly implemented to laboratory studies, because of all its advantages (reaction environment standardization, reagents, time and money savings etc.) [31, 32]. For this reason we decided to apply TMA-IHC methodology to check for possible associations between SATB1 protein expression with other markers and clinicopathological features.

\section{Material and methods}

Patients. The study was performed on material of 48 archival invasive ductal breast cancers (IDC), sampled in year 2000 during procedures of mammectomy in the Lower Silesian Oncology Centre in Wroclaw. All the patients were of female sex and grade of malignancy $\mathrm{G}$ was evaluated to be $\mathrm{G} 1$ in 10 cases, G2 in 28 cases and G3 in 10 cases (according to the 2003 World Health Organization (WHO) classification) [33]. The age of the patients ranged from 30 to 83 years (mean $56 \pm 11.1$ years).

Tissue microarray construction. For the construction of the tissue microarray blocks, a $7 \mu \mathrm{m}$ thick section was made from paraffin donor block and stained with hematoxylin and eosin (HE). The HE sections were examined by two independent pathologists under light microscope (BX-41,
Olympus, Tokyo, Japan) and areas of interest were circled using a permanent marker. From the corresponding paraffin blocks, $0.6 \mathrm{~mm}$ core punches were taken for each case using the Manual Tissue Arrayer I (Beecher Instruments Inc., Sun Praire, WI, USA) and transferred into the recipient paraffin block.

Immunohistochemistry. Immunohistochemical (IHC) reactions were performed on $4 \mu \mathrm{m}$ thick paraffin sections obtained from TMA blocks. The sections were dewaxed, re-hydrated and the epitopes were exposed using Pre-Treatment Link Rinse Station and Target Retrieval Solution (pH 6 for Ki-67, ER and PR; pH 9 for SATB1; $97^{\circ} \mathrm{C}, 20$ min) (DakoCytomation, Glostrup, Denmark). Activity of endogenous peroxidase was blocked by 5 min exposure to Peroxidase-Blocking Reagent (DakoCytomation). The sections were then rinsed with Wash Buffer and incubated for $20 \mathrm{~min}$ at room temperature with the following primary monoclonal antibodies against Ki-67 (clone MIB-1; Mouse; Ready-to-use; DakoCytomation), ER (clone 1D5; Mouse; Ready-to-use; DakoCytomation), PR (clone PgR 636; Mouse; Ready-to-use; DakoCytomation) and SATB1 (clone EPR3895; Rabbit; 1:100; Abcam, Cambridge, UK). Secondary goat antibodies coupled to a dextran core, linked to horseradish peroxidase, were applied and subsequent visualization was performed using the EnVision ${ }^{\mathrm{TM}}$ FLEX+ system (DakoCytomation) according to the manufacturer's instructions. All IHC reactions were performed in an automated staining platform, Autostainer Link48 (DakoCytomation). The reactions were visualized using 3,3'-diaminobenzidine tetrachlorohydrate (DAB+ Chromogen, DakoCytomation). All slides were counterstained with Mayer's hematoxylin.

Evaluation of the immunohistochemical reactions. For the evaluation of Ki-67 and SATB1 expression in each TMA core three fields with the highest number of tumor cells demonstrating positive reaction were selected (hot spots). The percentage of positive cells in each hot spot was evaluated under $\times 400$ magnification, scoring the brown-labeled cell nuclei of cancer cells (BX-41 light microscope equipped with Cell ${ }^{\mathrm{D}}$ software for computer-assisted image analysis; Olympus). The general result for every sample represented an average of the three hot spot evaluation. The status of estrogen and progesterone receptors expression was assessed according to their presence in the nuclei of cancer cells. If reaction was observed in over $10 \%$ of cancer cells it was classified as positive. The intensity of the IHC reactions in coded TMAs was independently evaluated by two pathologists. Moreover, in doubtful cases, re-evaluation with a double-headed microscope was performed until a consensus was achieved.

Statistical analysis. The results were subjected to statistical analysis using Prism 6.0 software (GraphPad, La Jolla, CA, USA). The relationship between the expressions of Ki-67 

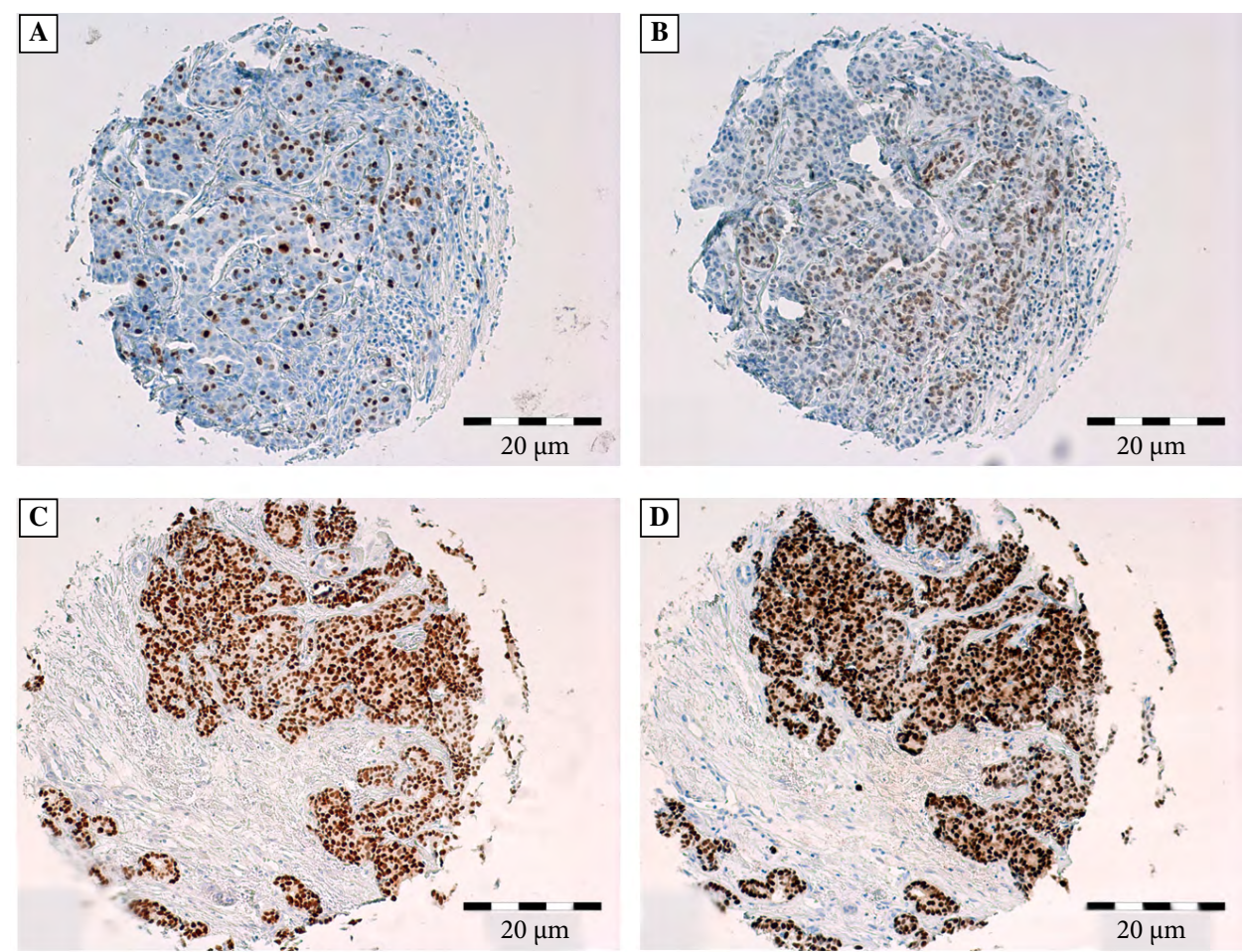

Figure 1. Nuclear expression of Ki-67 (A), SATB1 (B), ER (C), PR (D) on serial sections of invasive ductal breast carcinoma. Immunohistochemistry performed on tissue microarrays (TMA-IHC method); magnification $\times 100$

and SATB1 in regard to hormone receptor status was examined using Spearman's rank correlation test. The associations between expression intensities of analyzed markers with histological malignancy grade (G) were examined by Kruskal-Wallis and Mann-Whitney tests. In all analyses, results were considered to be statistically significant when $p<0.05$.

\section{Results}

IHC reactions were localized in nuclear compartment of the cancer cells. Expression of Ki-67 and SATB1 protein was observed in $89.58 \%$ and $31.25 \%$ of IDCs, respectively. $62.5 \%$ of tumors were classified as ER -positive, and 47.92\% as PR-positive. Analysis of Ki67, SATB1, ER and PR expression was conducted on serial sections (Figure 1A, B, C, and D, respectively). The correlations between expression of the studied proteins were found to be moderate $(\mathrm{r}=0.291$, $p=0.045$ independently on the receptor status, and $\mathrm{r}=0.392, p=0.032$ in ER-negative tumors; Spearman's correlation test). All correlation results are shown in Table 1. The expression of the Ki-67 antigen increased with higher grade of histological malignancy. Statistically significant differences were found between: G1 vs. G2 $(p=0.0002), \mathrm{G} 2$ vs. G3 $(p=0.0253)$, and G1 vs. G3 ( $p=0.0184)$ (Mann-Whitney test; Figure 2). Similar trend was noted in regard to SATB1 protein expression, but statistical signifi- cance level has not been reached (Mann-Whitney and Kruskal-Wallis tests; Figure 3).

\section{Discussion}

Cancer progression and metastasis involve series of alterations in the expression of multitude of genes. The structure and organization of chromatin play an important role in spatial arrangement of genes inside cell nucleus thereby allowing different mechanisms to activate or silence the transcription of genes governed by various epigenetic events. Epigenetic modifications and dynamic changes in chromatin organization by organizer proteins have recently been shown to play an instrumental role in regulating cancer-promoting genes [34, 35]. Aberrant expression of SATB1 mRNA and protein has been shown to promote growth and metastasis of various neoplasms [10-26]. The main and most quoted role of SATB1 is chromatin organization and functioning as a global regulator of gene expression, among others during cancer development (e.g. it directly regulates the expression of ERRB2, MMP2, ABL1, and E-cadherin) [34, 36]. The expression of SATB1 progressively increases with the progression of cancer and it was suggested that SATB1 dynamically reprograms the expression of genes that are involved in carcinogenesis, e.g. by determining specific epigenetic modifications at target gene loci 
Table 1. Correlation of expression intensities of analyzed markers: Ki-67 vs. SATB1 in regard to receptor status; Spearman's correlation test

\begin{tabular}{|l|l|l|}
\hline $\begin{array}{l}\text { Correlation according } \\
\text { to receptor status }\end{array}$ & $\mathbf{R}$ & $\boldsymbol{p}$ \\
\hline All tumors & $\mathbf{0 . 2 9 1}$ & $\mathbf{0 . 0 4 5}$ \\
\hline ER - positive tumors & 0.124 & NS \\
\hline ER - negative tumors & $\mathbf{0 . 3 9 2}$ & $\mathbf{0 . 0 3 2}$ \\
\hline PR - positive tumors & 0.133 & NS \\
\hline PR - negative tumors & 0.366 & NS \\
\hline
\end{tabular}

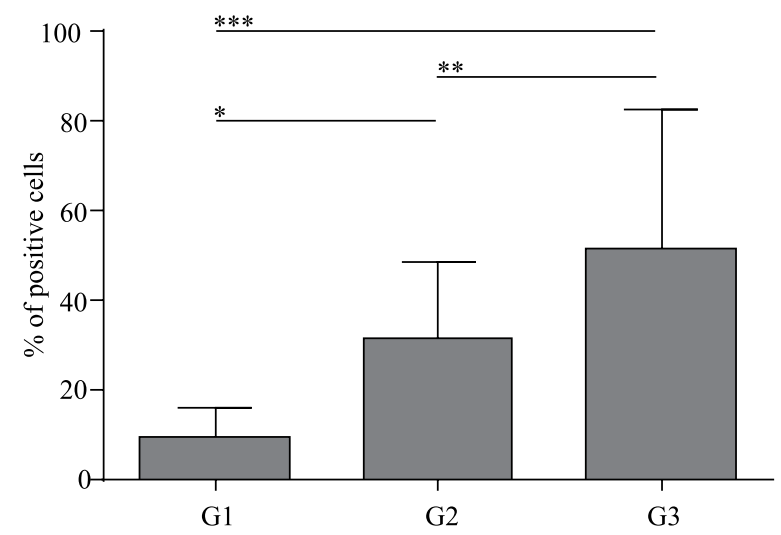

Figure 2. Expression of Ki-67 according to histological grade of malignancy $(\mathrm{G}) .{ }^{*} \mathrm{G} 1$ vs. G2 $(p=0.0002),{ }^{* *} \mathrm{G} 2$ vs. G3 $(p=0.0253),{ }^{* *} \mathrm{G} 1$ vs. G3 $(p=0.0184)$; Mann-Whitney test

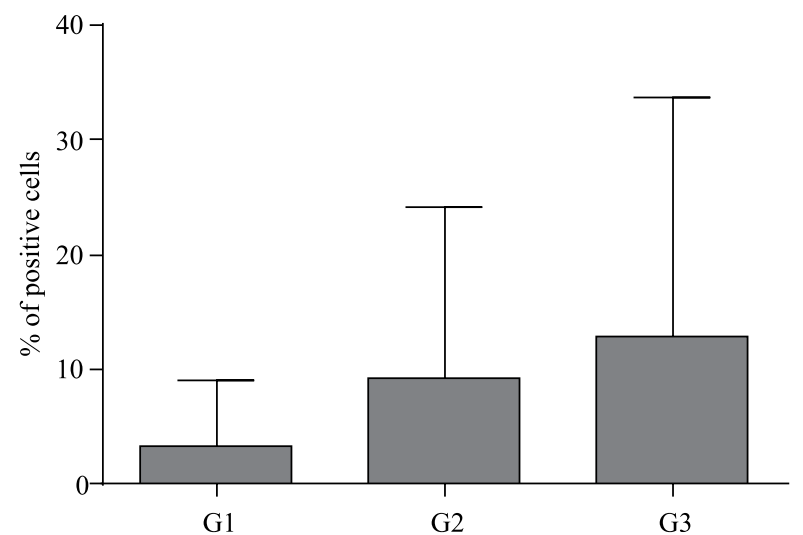

Figure 3. Expression of SATB1 according to histological grade of malignancy $(\mathrm{G})$ was statistically insignificant

and directly up-regulating metastasis-associated genes while down-regulating tumor-suppressor genes [34]. As SATB1 reprograms chromatin organization and the transcription profiles to promote growth and metastasis in breast cancer, it may be presumed that mutation of the $S A T B 1$ gene might affect not only cell cycle progression but also apoptosis pathway in breast cancer [34].
In our study we checked the association between SATB1 protein expression with well-known marker of proliferation - Ki-67 antigen in relation to ER and PR status. The moderate correlation between analyzed markers independently to the receptor status found in our study in our opinion indicates the indirect role of this protein in the cancer cell proliferation. This is concomitant with results of other studies on SATB1 expression in cancers. E.g. Laurinavicius et al. [30] carried out IHC studies on tissue microarrays of 109 patients with ductal breast carcinoma and stained a set of 10 IHC markers - ER, PR, HER2, Ki67, AR, BCL2, HIF-1 $\alpha$, SATB1, p53, and p16. They did not observe any significant associations between SATB1 and other tested markers, except HIF-1 $\alpha$. The authors focused on clinical application of results, so they analyzed expression of markers according to the status of hormone. They implied that SATB1 and HIF- $1 \alpha$ may be important markers of the disease associated with ER-positive cancers, whereas their biological and clinical significance remains to be elucidated [30]. Their findings seem to be contrary to our data since we found moderate correlation between expression of SATB1 and Ki-67 in ER-negative tumors. Similar results were shown by Patani et al. who found that high SATB1 expression levels occurred more often in ERnegative cancers [29]. Towards to this observations, the use of SATB1 as target or prognostic marker for breast cancer should be viewed with caution and a possible confounding effect of the estrogen receptor status of the tumor should be taken into account when analyzing the diagnostic and prognostic value of SATB1 [30, 37].

We additionally checked relationships between expressions of tested markers and grade of histological malignancy $(\mathrm{G})$. Stronger expression of SATB1 and $\mathrm{Ki}-67$ proteins was seen in higher grades of malignancy, but only Ki-67 association reached statistical significance level. Ki-67 expression confirms the correct choice of the studied group, whereas in case of SATB1 vs. G relation, similar results were observed in literature [30].

The very interesting property of SATB1, pointing at still not fully explored role of this protein in carcinogenesis, was described Han et al. [38]. They showed that SATB1 was expressed by aggressive breast cancer cells and it had high prognostic significance, independently on lymph-node status. After knockdown of SATB1 in highly aggressive (MDA-MB-231) cancer cell line they observed a shift in the expression of over 1000 genes, reversal of tumorigenesis by restoring breast-like acinar cell polarity and inhibition of tumor growth and metastasis. Moreover, the ectopic SATB1 expression in non-aggressive (SKBR3) cells led to gene expression patterns consistent with aggres- 
sive-tumor phenotypes, acquiring metastatic activity [38]. However, these results were not confirmed by Iorns et al. [39] who conducted similar, very precise study, using the same breast cancer cell lines. None of the results obtained by Han et al. was reached, what highly limits drawing direct conclusions [39].

In summary, the evaluation of the SATB1 protein expression could be very useful for cancer diagnostics and treatment purposes if its relationships with other cellular proteins were further and better characterized. Our results, showing moderate correlation of SATB1 with Ki-67, especially in ER-negative IDCs suggest rather indirect role of this protein in cancer cell proliferation. Statistically insignificant association of SATB1 with grade of histological malignancy in relation to contrary results achieved by other authors indicate a need for further studies before drawing particular conclusions. Furthermore, understanding the molecular mechanisms of the regulation of SATB1 expression would therefore be essential towards designing strategies to control it.

\section{Acknowledgements}

The Authors thank Dr. Aleksandra Piotrowska and Mrs. Karolina Jablonska from the Department of Histology and Embryology, Wroclaw Medical University, Poland, for their technical support. The research was supported by Wroclaw Research Centre EIT+ under the project "Biotechnologies and advanced medical technologies" - BioMed (POIG.01.01.0202-003/08), financed by the European Regional Development Fund (Operational Programme Innovative Economy, 1.1.2).

\section{References}

1. Mirkovitch J, Mirault ME, Laemmli UK. Organization of the higher-order chromatin loop: specific DNA attachment sites on nuclear scaffold. Cell. 1984;39:223-232.

2. Nelson WG, Pienta KJ, Barrack ER et al. The role of the nuclear matrix in the organization and function of DNA. Annu Rev Biophys Biophys Chem. 1986;15:457-475.

3. Galande S. Chromatin (dis)organization and cancer: BUR-binding proteins as biomarkers for cancer. Curr Cancer Drug Targets. 2002;2:157-190.

4. Boulikas T. Chromatin domains and prediction of MAR sequences. Int Rev Cytol. 1995;162A:279-388.

5. Yasui D, Miyano M, Cai S et al. SATB1 targets chromatin remodelling to regulate genes over long distances. Nature. 2002;10:641-645.

6. Galande S, Purbey PK, Notani D et al. The third dimension of gene regulation: organization of dynamic chromatin loopscape by SATB1. Curr Opin Genet Dev. 2007;17:408-414.

7. Kohwi-Shigematsu T, Poterlowicz K, Ordinario E et al. Genome organizing function of SATB1 in tumor progression. Semin Cancer Biol. 2013;23:72-79.

8. Cai S, Han HJ, Kohwi-Shigematsu T. Tissue-specific nuclear architecture and gene expression regulated by SATB1. Nat Genet. 2003;34:42-51.
9. Kim CJ, Lee GR, Shin JW et al. Mutational analysis of SATB1 gene in hepatocellular carcinomas. APMIS. 2013;121:10121014.

10. Zheng J. Is SATB1 a master regulator in breast cancer growth and metastasis? Womens Health (Lond Engl). 2008;4:329-332.

11. Cheng C, Lu X, Wang G et al. Expression of SATB1 and heparanase in gastric cancer and its relationship to clinicopathologic features. APMIS. 2010;118:855-863.

12. Fang XF, Hou ZB, Dai XZ et al. Special AT-rich sequence-binding protein 1 promotes cell growth and metastasis in colorectal cancer. World J Gastroenterol. 2013;19:2331-2339.

13. Kapoor S. SATB1 expression and its association with tumour prognosis in systemic malignancies: an evolving concept in oncology. Liver Int. 2013;33:322.

14. Zhao XD, Ji WY, Zhang W et al. Overexpression of SATB1 in laryngeal squamous cell carcinoma. ORL J Otorhinolaryngol Relat Spec. 2010;72:1-5.

15. Selinger CI, Cooper WA, Al-Sohaily S et al. Loss of special AT-rich binding protein 1 expression is a marker of poor survival in lung cancer. J Thorac Oncol. 2011;7:1179-1189.

16. Huang B, Zhou H, Wang $X$ et al. Silencing SATB1 with siRNA inhibits the proliferation and invasion of small cell lung cancer cells. Cancer Cell Int. 2013;13:8.

17. Murono S, Tsuji A, Endo K et al. Immunohistochemical detection of SATB1 is independent of thyroid cancer differentiation. Laryngoscope. 2013;123:2909-2912.

18. Han B, Luan L, Xu Z et al. Expression and biological roles of SATB1 in human bladder cancer. Tumour Biol. 2013;34:2943-2949.

19. Nodin B, Hedner C, Uhlén M et al. Expression of the global regulator SATB1 is an independent factor of poor prognosis in high grade epithelial ovarian cancer.J Ovarian Res. 2012;5:24.

20. Xiang J, Zhou L, Li S et al. AT-rich sequence binding protein 1: Contribution to tumor progression and metastasis of human ovarian carcinoma. Oncol Lett. 2012;4:865-870.

21. Mao L, Yang C, Wang J et al. SATB1 is overexpressed in metastatic prostate cancer and promotes prostate cancer cell growth and invasion. J Transl Med. 2013;11:111.

22. Chen H, Takahara M, Oba $\mathbf{J}$ et al. Clinicopathologic and prognostic significance of SATB1 in cutaneous malignant melanoma. J Dermatol Sci. 2011;64:39-44.

23. Zhang H, Qu S, Li S et al. Silencing SATB1 inhibits proliferation of human osteosarcoma U2OS cells. Mol Cell Biochem. 2013;378:39-45.

24. Chu SH, Ma YB, Feng DF et al. Upregulation of SATB1 is associated with the development and progression of glioma. J Transl Med. 2012;10:149.

25. Han S, Xia J, Qin X et al. Phosphorylated SATB1 is associated with the progression and prognosis of glioma. Cell Death Dis. 2013;4:e901.

26. Nakayama Y, Mian IS, Kohwi-Shigematsu T et al. A nuclear targeting determinant for SATB1, a genome organizer in the T cell lineage. Cell Cycle. 2005;4:1099-1106.

27. Nodin B, Johannesson H, Wangefjord S et al. Molecular correlates and prognostic significance of SATB1 expression in colorectal cancer. Diagn Pathol. 2012;7:115.

28. Meng WJ, Yan H, Zhou B et al. Correlation of SATB1 overexpression with the progression of human rectal cancer. Int J Colorectal Dis. 2012;27:143-150.

29. Patani N, Jiang W, Mansel R et al. The mRNA expression of SATB1 and SATB2 in human breast cancer. Cancer Cell Int. 2009;9:18.

30. Laurinavicius A, Laurinaviciene A, Ostapenko V et al. Immunohistochemistry profiles of breast ductal carcinoma: factor analysis of digital image analysis data. Diagn Pathol. 2012;7:27. 
31. Kobierzycki C, Malinska A., Dziegiel P. Application of tissue microarray technique (TMA) in neoplastic markers studies on breast cancers. Adv Cell Biol. 2012;39:519-530.

32. Kobierzycki C, Pula B, Wojnar A et al. Tissue microarray technique in evaluation of proliferative activity in invasive ductal breast cancer. Anticancer Res. 2012;32:773-777 .

33. Tavassoli FA and Devilee P. Pathology and Genetics of Tumours of the Breast and Female Genital Organs. World Health Organization Classification of Tumours. IARC Press, Lyon, 2003.

34. Mir R, Pradhan SJ, Galande S. Chromatin organizer SATB1 as a novel molecular target for cancer therapy. Curr Drug Targets. 2012;13:1603-1615.
35. Cai S, Lee CC, Kohwi-Shigematsu T. SATB1 packages densely looped, transcriptionally active chromatin for coordinated expression of cytokine genes. Nat Genet. 2006;38:1278-1288.

36. Pavan Kumar P, Purbey PK, Sinha CK et al. Phosphorylation of SATB1, a global gene regulator, acts as a molecular switch regulating its transcriptional activity in vivo. Mol Cell. 2006;22:231-243.

37. Hanker LC, Karn T, Mavrova-Risteska L et al. SATB1 gene expression and breast cancer prognosis. Breast. 2011;20: 309-313.

38. Han HJ, Russo J, Kohwi Y et al. SATB1 reprogrammes gene expression to promote breast tumour growth and metastasis. Nature. 2008;7184:187-193.

39. Iorns E, Hnatyszyn HJ, Seo P et al. The role of SATB1 in breast cancer pathogenesis. J Natl Cancer Inst. 2010;102:1284-1296.

Submitted: 11 January, 2014 Accepted after reviews: 18 January, 2014 